\title{
GOETHE UND DIE KUSTENVERANDERUNGEN BEI NEAPEL
}

\author{
VON LEONHARD Franz
}

Mit einer Abbildung

Im Jahre 1823 behandelte GOETHE in einem "Architektonisch-naturhistorisches Problem» betitelten Beitrage zu seinen naturhistorischen Schriften ${ }^{1}$ eine Erscheinung an den Marmorsäulen des antiken Serapäums von Pozzuoli, dem altrömischen Puteoli, westlich von Neapel, die so merkwürdig ist, daß heute noch der Baedeker auf sie aufmerksam macht. Der mittlere Teil der drei noch stehenden Säulen des 1752 als Steinbruch aus alter Verschüttung wiederausgegrabenen Bauwerkes ist in einer Höhe von 3,60 bis $5,70 \mathrm{~m}$ über dem Boden von Bohrmuscheln (Lithodomus lithophagus) angebohrt. Daraus ist der Schluß gezogen worden, daß das Meer diese Gegend einmal überflutet hat, und zwar so, daß die Muscheln ihre Bohrtätigkeit in den Säulen ziemlich hoch über deren Basis ausüben konnten.

Diesen Weg zur Erklärung des Phänomens an den Säulen hat zum Beispiel der Altertumsforscher FrIEDR. SICKLER in einer Abhandlung in Bd. 5 (1816) der von GOETHES Schwager VulPIUs herausgegebenen Zeitschrift «Curiositäten» eingeschlagen². SICKLER schrieb da:

"Als der an den Berg, an dem Pozzuolo größtenteils liegt, mit dem Rücken anstoßende Tempel aufgegraben ward, fand man im eingestürzten Ruinenschutt von höher liegenden, alten, ehemaligen Gebäuden zuerst die Spitzen der drei großen Säulen. Darauf fand man den Ort, wo die Bohrmuscheln sich eingearbeitet hatten, und von da an, wo dieser aufhörte, grub man den Úberrest der Säulen nebst der ganzen Basis des Gebäudes nicht aus den Ruinen, sondern aus Berg- und Felsenschutt aus. Hierauf gründet man nun folgende Erklärung. Als der Berg von Pozzuolo, sei es durch Erdbeben oder durch Meeresfluten erschüttert, das alte Gebäude überdeckte, reichte der Schutt bis an die Mitte der drei stehend gebliebenen Säulen, und dieser bildete für das (wahrscheinlich in demselben Ereignisse) höher gestiegene Meer den Meeresgrund, wodurch es möglich ward, da $\beta$ die Bohrmuscheln sich gegen drei bis vier Fuß an den Säulen ansetzen konnten, da das Meer nur so hoch über dem Schuttgrund stand. Über der Oberfläche blieben folglich die Spitzen der Säulen hervorragend, woran die Bohrmuscheln nicht kommen konnten. Bei einer späteren Revolution wurden auch diese überdeckt; das Meer zog sich wieder zurück, und in diesem Zustand ward nun das uralte Gebäude gefunden. ... Mag nun auch der mit dem Gegenstand genau bekannte und an Ort und Stelle vertraut gewordene Altertumsforscher diese Erklärung annehmen oder nicht, so bleibt doch so viel gewiß, daß die Stelle, wo die Pholaden sich angesetzt, bis auf 18-20 Fuß über der Meeresoberfläche sich erhebt, folglich mit allen Felsen an der Küste Italiens, die von Pholaden angefressen sind, in derselben Höhe steht.»

"Das zweite dieser Monumente», fährt Sickler fort, "sind die drei Tempel zu Pästum. Diese ... bieten dieselbe Erscheinung dar. Auch sie sind durchgängig von den Bohrmuscheln angefressen, und die ehrwürdigsten und majestätisch kolossalen Säulen ... erblickt man bis zu vier und acht Fuß von der Basis an überall durchbohrt ... Der obere Teil der Säulen ist ganz frei von diesem Pholadenfraß, so weit nämlich, als er aus dem Meere emporragte, und auch in diesen Löchern werden oft und habe ich selbst Bohrmuscheln verwittert gefunden. Ubrigens mag auch hier die Höhe, bis zu der die Pholaden gereicht, weit mehr oder weniger als $20 \mathrm{Fu} ß$ über der jetzigen Meereshöhe betragen. Und so fände sich denn auch hierin Übereinstimmung mit den Säulen zu Pozzuolo usw. In getreu darstellenden Kupfern sind diese Pholadenverwüstungen, die man bisher für Beschädigungen durch die dort weidenden Viehherden hielt, ebenfalls gewöhnlich angegeben.»

Auf die Säulen von Pozzuoli kommt auch K. E. A. von Hoff in seiner "Geschichte der durch Überlieferung nachgewiesenen natürlichen Veränderungen der Erdoberfläche» zu sprechen ${ }^{3}$, wobei er die Abhandlung Sicklers und andere Autoren zitiert. Hoff

1 Weimarer Ausgabe, II. Abt., Bd. 10, S. 191.

2 Der langatmige Titel der Abhandlung lautet: Úber das Eindringen des Meeres in Italiens Ebenen zur Zeit der Blüte der älteren euböischen, dorischen und achäischen Kolonien in Groß-Griechenland und über die muthmaßliche Entstehungsperiode der kampanischen Vasen.

31. Teil (Gotha 1822), S. 455. 
erklärt sich außerstande, SickLERs Erklärung annehmen zu können, ohne jedoch selbst eine Erklärung zu bieten. Er gibt sogar zu erwägen, ob die Säulen etwa aus Blöcken hergestellt seien, die schon in ihrer natürlichen Lagerstätte von Muscheln angebohrt waren.

1787 hatte GoEthe die UUberreste des Serapäums in Pozzuoli betrachtet und am 19. Mai in seinem Tagebuche einen Gedankengang notiert, den er 1823 in seinen naturwissenschaftlichen Schriften wieder aufnahm. Zu dem Aufsatz hat ihn, wie er in diesem mitteilt, zweierlei bewogen, nämlich einerseits, daß ihm ein Baumeister drei Zeichnungen zur Deutung des Problems herstellte (hier S. 209), anderseits, daß HofF des Falles gedachte.

Wie aus GoEthes Tagebuchaufzeichnungen vom 19. Mai 1787 hervorgeht, hat er sich damals selbst eine Skizze angefertigt, auf der er die verschiedenen Niveauhöhen der Tempelruinen mit Buchstaben bezeichnete. Diese Skizze war sicherlich die Grundlage für die spätere Zeichnung. In dem Aufsatz von 1823 nennt Goethe zwar den Stecher der Tafel, den Weimarer Kupferstecher Karl Aug. Schwerdgeburth (1785 bis 1878) ${ }^{4}$, aber nicht den Zeichner. Er bezeichnet diesen als Baumeister, in seinem Briefe vom 9. Februar 1823 an Hoff, der hier S. 208 abgedruckt ist, als Architekten, wobei GoEtHe erwähnt, daß die Tafel «schon einige Jahre» unter seinen (GoETHEs) Papieren gelegen sei; daraus ergibt sich, daß sie nicht erst 1823 angefertigt wurde.

Zum 9. Februar 1823 steht in Goethes Tagebuch: «... Kupferwerke wegen des Tempels zu Puzzuol»; Goethe hat also damals die Antichità di Puzzuolo des P. ANt. PaOlI und die Description des royaumes de Naples et Sicile studiert, die er als Bilderquellen zum Serapäum auch in seinem Aufsatz 1823 anführt. Dort sagt er, daß die 15. Tafel in dem italienischen Werke den damaligen Zustand des Pozzuoli-Tempels ungefähr so zeigt wie eine in der Weimarer Bibliothek verwahrte, durch den Maler und Baumeister MaximILIAN von Verschaffelt (1754-1818) 1790 hergestellte Zeichnung. Auf Studium dieser Zeichnung ist offenbar GoETHES Tagebucheintragung am 13. Februar $1823 \mathrm{zu}$ beziehen: «... Landschaftliche Zeichnung der angefressenen Marmorsäulen von Verschaffelt ...» Laut Tagebuch hatte Goethe dann am 16. Februar mit "Oberbaudirector Coudray Verabredung wegen des Tempels zu Puzzuol». Coudray sollte anscheinend einen Abdruck der Kupferplatte Schwerdgeburths nach der von GoETHE vor Jahren besorgten Zeichnung der Tempelruinen verschaffen; denn im Tagebuche heißt es unterm 15. April 1823: «... Oberbaudirector CoudraY, welcher das Kupfer vom Tempel des Serapis zu sich nahm ... ., am 18. April: «... Herr Oberbaudirector Coudray, einen Abdruck des Kupfers vom Tempel zu Pozzuol bringend ...."

Wer der Zeichner gewesen, bleibt mithin unsicher; vielleicht war es C. CoudraY (1775-1845), der seit 1815 in Weimar tätig war. Jedenfalls beweisen die angeführten Tagebuchnotizen, daß GoETHE die Angelegenheit des Serapäums nicht aus dem Ärmel geschüttelt, sondern sorgfältig verfolgt hat. Am 27. April 1823 ist er laut Tagebuch mit RIEMER "den puzzuolischen Tempel durchgegangen», er hat also mit seinem Freunde das Problem besprochen, anscheinend knapp vor oder nach der Niederschrift des Aufsätzleins.

Das Werk Hofrs, das nach GoEthes eigenem Zeugnis den Aufsatz letzten Endes hervorgerufen hat, findet sich in GoETHEs Schriften einige Male erwähnt. In den "Annalen» 1822 nennt er es "einen Schatz, zu welchem man immer etwas hinzutun möchte, indem man sich daran bereichert", und er erwähnt, daß es ihm bei seinen naturwissenschaftlichen Bemühungen "neuen Reiz» gegeben habe. "Herrn voN HofFs geologischem Werk» ist ein eigener Beitrag in GOETHES naturwissenschaftlichen Schriften gewidmet.

4 In Goethes Tagebuch ist zum 25. April 1823 vermerkt: «. . . Schwerdgeburth wegen der Tafel zum Tempel ... .», drei Tage später: «. . . Schwerdgeburth Probedruck des puzzuolischen Tempels...» 
Am 9. Februar 1823 schrieb GoEthe an Hoff, daß er dessen Werke «einiges Einzelne bekräftigend erwidern" wolle und daß er in seinen Papieren "eine ganz befriedigende Auflösung des Rätseltempels zu Puzzuol" gefunden habe.

«Die Blätter datieren sich Neapel, Sonnabend den 19. May 1787, also nach meiner Rückkehr von Sicilien; ich ließ beym Abdruck meiner Reisebeschreibung diese Stelle weg, weil ich ein Kupfer dazu nothwendig fand. Die von einem Architekten deshalb entworfene Tafel liegt schon einige Jahre unter anderen Papieren und wäre ohne Ihre Anregung vielleicht verlorengegangen. Es läßt sich diese Erscheinung gar wohl örtlich deuten, ohne daß man das Mittelmeer, seit den Zeiten Diocletrans, etliche und dreyßig Fuß über sein Niveau bey Puzzuol zu bemühen braucht. Wunderlich genug, daß gewisse Köpfe solche desperate Erklärungsweisen für ganz bequem und natürlich finden. Ich müßte den übrigen Raum dieses Blatts mit Austufungszeichen füllen, wenn ich meine Gesinnungen über die desperaten Erklärungsweisen auszudrücken versuchte, womit bald ganze Reiche erhoben, bald das Meer aufsteigend, bald Continente zum Versinken verdammt werden; ist mir's doch, als wenn Neptun und Pluto nach Christi Geburth miteinander wetteiferten. Ew. Hochwohlgeboren haben das große Verdienst, diese Thorheiten mit größter Mäßigung zur Sprache zu bringen, und es muß ein jeder Sinnige für Pflicht halten, sich an Sie anzuschließen...»

Gozthe hat sich also als Feind der Annahme besonders gewaltsamer und weitreichender Naturumwälzungen in historischer Zeit ausgesprochen und beim Serapäum-Problem das Gewicht auf eine möglichst wenig gewaltsame und rein örtliche Erklärung gelegt. Seine Empörung gegenüber den «desperaten» Erklärern, unter die er wohl auch SrCKLER gerechnet haben muß, geht aus einer (undatierten) Notiz «Tempel zu Puzzol» ebenfalls hervor ${ }^{5}$ :

«Merkwürdiger ist nichts in der Welt, als daß man, um Phänomene zu erklären, die gewaltsamsten Mittel zu Hilfe ruft, anstatt daß man bey ruhiger Umsicht das nächste Natürliche bey der Hand gehabt hätte. So wie nun ein mächtiger Geolog, dem übernatürliche Hebel zu Gebote stehen, Seite 447 Schweden und Norwegen ohne Bedenken aus der Tiefe in die Höhe hebt und durch dieses desperate Mittel sich aus einer gewissen Verlegenheit zu helfen sucht, so tritt Seite 457 ein anderer auf, der den mächtigsten Damm durchsticht, so daß die Ufer des Mittelländischen Meeres dreyßig Fuß, und zwar auf eine Zeitlang unter Wasser gesetzt werden ${ }^{6}$. Hievon soll nun der Tempel zu Puzzol Zeugniß geben.

Dieser Tempel ist zu Drocletrans Zeiten gebaut worden; schon dies hätte einem kunstverständigen Naturforscher sagen sollen, daß nach dem Jahre das Meer weder so hoch, weder so lange in dieser Höhe habe stehen können, aber der mechanischen Erklärung ist nichts zu absurd, was sie nicht ganz natürlich fände. Doch wir wollen Schritt für Schritt vorwärtsgehen.»

Die einen Erklärer des Pozzuoli-Problems argumentierten nach Goethes eigenen Worten im Aufsatz von 1823 folgendermaßen: "Die Säulen, sagte man, sind von Pholaden angefressen; diese leben nur im Meere, das Meer muß also so hoch gestiegen und die Säulen ein Zeitlang von ihm umgeben worden sein.»

GoEthes Gedankengang hingegen war der: "Eine solche Schlußfolge darf man nur umkehren und sagen: eben weil man die Wirkung der Pholaden hier mehr als dreißig Fuß über dem Meeresspiegel findet und sich ein zufälliger Teich hier oben nachweisen läßt, so müssen Pholaden, von welcher Art sie auch seien, im süßen oder doch durch vulkanische Asche angesalzten Wasser existieren können. Und hier spreche ich im allgemeinen unbedenklich aus: eine Erklärung, die sich auf eine neue Erfahrung stützt, ist achtungswert.»

Zur Unterstützung seiner Auffassung verweist GoEthe darauf, daß eine Erhöhung des Mittelmeeres im Mittelalter doch in irgendeiner Chronik festgehalten worden sein

5 Weimarer Ausgabe, II. Abt., Bd. 10, S. 255.

${ }^{6}$ Die beiden Seitenangaben in Goethes Notiz beziehen sich auf HoFfs hier Seite 206 genanntes Werk, und zwar auf dessen 1. Teil, der 1822 erschienen ist, wodurch für die Notiz ein terminus ante quem non gegeben ist. Auf Seite 447 spricht Hoff sich gegen die Annahme Leopold von Buchs aus, $\mathrm{da}$ sich die schwedische Landmasse langsam hebe, und er nennt das ein "wahrhaft desperates Mittel der Erklärung». GoEthe hat also den in seinem Briefe an HoFf und in der Notiz verwendeten Ausdruck "desperate Erklärungsweisen» von Hoff übernommen. Auf Seite 457 ist die Rede von der durch SickLER aufgegriffenen Annahme eines Durchbruchs des Bosporus zu einer Zeit, in der die Straße von Gibraltar noch nicht bestanden habe, ferner davon, daß «der erstere dieser Durchbrüche ... eine so große Überflutung der Küstenländer, als zur Erklärung der Erscheinung bei Pozzuolo erforderlich gewesen sein würde, wohl nicht hervorgebracht haben könnte». 
würde, was aber nicht der Fall sei ${ }^{7}$. Er erwähnt sodann den möglichen Einwand, $\mathrm{da} B$ die Erhöhung des Meeresniveaus nicht während unserer christlichen Zeitrechnung eingetreten sei, sondern "frühern Jahren, vielleicht gar dem poetischen Kreise " angehört habe. Dazu bemerkt er in zutreffender Selbstcharakterisierung, $\mathrm{da} B$ er Streit und Widerstreit nicht liebe; «für uns ist's genug, daß ein Tempel, im dritten Jahrhundert erbaut, wohl schwerlich in dem Maße vom Meere jemals überschwemmt worden sein" könne. Mit der Bemerkung von dem poetischen Kreise hat GOETHE vielleicht auf Sickler gezielt, der die Überflutung in die Zeit des Pythagoras (6. Jahrh. v. Chr.) datiert. Gegen diese Datierung führt GoETHE mit Recht das geringere Alter des Serapäums an, wobei er freilich zu niedrig gegriffen hat; denn eine "aedes Serapis" ist inschriftlich für Puteoli schon 105 v. Chr. bezeugt, nicht erst aus der Zeit des Kaisers Dicletianus (284 bis 305 n. Chr.) ${ }^{8}$. Durch

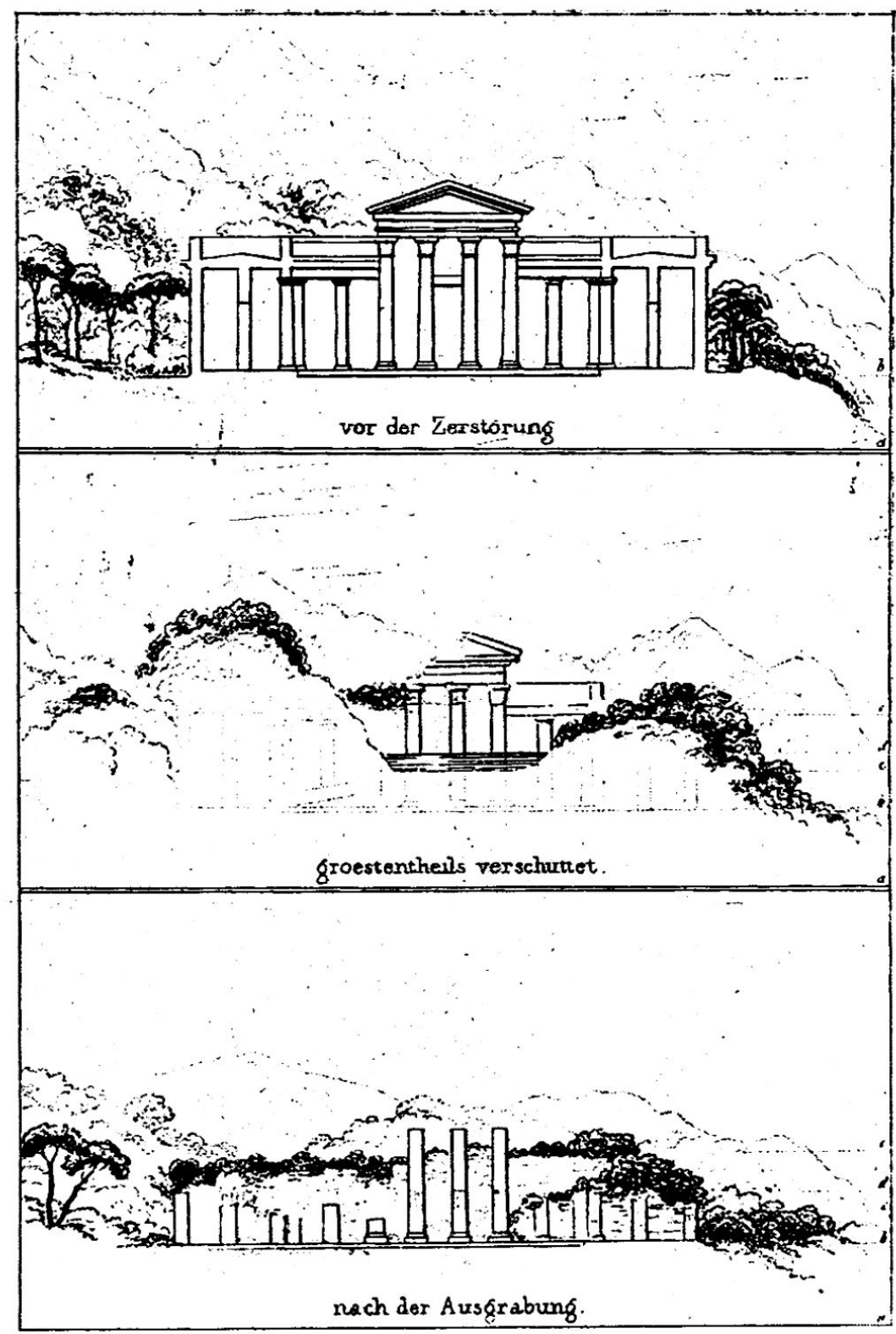

Schematische Darstellung zur Erklärung des Pozzuoli-Problems, nach Goethes Angaben gezeichnet.

\section{Goerhes Erklärung des Phä-}

nomens von Puteoli oder vielleicht mehr durch die Tatsache, daß es Goethe war, der sie gegeben hat, war Hoff so beeindruckt, daß er sie im 2. Teil seines Werkes als "naturgemäß und aufklärend» anerkannte", wobei er hinzufügte, daß schon die

7 Vielleicht bezieht sich darauf, was Hoff am 7. Jụni 1823 an GoEthe geschrieben hat: "Nun ist die Verschüttung, welche Ew. Excellenz für den Serapistempel annehmen, und deren es bedarf, noch gar nicht von solcher Größe, daß sie ein großes Aufsehen in der Welt hätte erregen müssen. Sie ist noch lange kein Monte nuovo, und es mögen leicht manche Veränderungen in der äußeren Bodengestalt der Campi phlegraei in jenen Zeiträumen vorgegangen sein, zu denen uns die historischen Data fehlen und immer fehlen werden.» Den Monte nuovo nordwestlich Pozzuoli, der 1538 vulkanisch emporgewachsen ist, hatte GoETHE für die Verschüttung des Tempels in Anspruch genommen. Auf die Phlegräischen Felder wurde von ihm gleichfalls, als Ursache ständiger Bodenbewegungen, hingewiesen; sie sind bekanntlich das vulkanische Gebiet nördlich von Pozzuoli mit alten Kratern und Gasquellen; ebenso ist die von GoETHE herangezogene Solfatara nordöstlich von Pozzuoli eine vulkanische Schwefelgasquelle.

${ }^{8}$ Literatur über Puteoli und über das Serapäum ist verzeichnet bei MaU-MerckLIN: Katalog der Bibliothek des Deutschen Archäologischen Institutes in Rom, I/1, S. $492 \mathrm{ff}$.

๑ Gotha 1824, S. 203. 
Italiener Piñ und Brocchi eine im wesentlichen mit GoEthes Vorstellung gleiche Ansicht geäußert hatten.

Wenn das Phänomen vereinzelt wäre, erschiene GoEthes Erklärung in der Tat als annehmbar, und man müßte dann auch seinem Schlusse auf die Lebensbedingungen der Bohrmuscheln folgen, obschon Lithodomus sonst nur in Salzwasser festgestellt ist.

Aber das Phänomen ist nicht vereinzelt. Schon SICKLER hat darauf hingewiesen, daß die Stelle, wo an den Säulen die Muscheln sich angesetzt hatten, "mit allen Felsen an der Küste Italiens, die von Muscheln angefressen sind, in derselben Höhe stehen» und daß das gleiche auch von den Tempeln zu Pästum gilt. Die Erklärung, die Goethe für Puteoli gegeben hat, kann nicht auch für die letztgenannten Tempel gelten, weil dort die Voraussetzungen für das Entstehen einer Lagune nicht vorhanden waren. Die Muschellöcher sind also doch nur durch eine zeitweilige Änderung der Lage des Meeresspiegels verständlich, wobei jedoch nicht das Land unbewegt geblieben und das Meer gestiegen ist, sondern es handelt sich um eine Landsenkung und spätere neuerliche Hebung.

GOETHE hat von der, seiner eigenen Auffassung entgegenstehenden Erklärung gewußt, wie aus seinen Äußerungen klar hervorgeht. Er muß auch davon gewußt haben, $\mathrm{da} ß$ es Muschellöcher anderwärts gibt; mindestens konnte er das aus SICKLERs Abhandlung erfahren. Es ist kaum anzunehmen, daß er diese nicht gelesen habe, ist sie doch in einer Zeitschrift gedruckt, die an Goethes Wohnort erschienen und von seinem eigenen Schwager herausgegeben worden ist, in einer Zeitschrift, an der GOETHE gelegentlich selbst mitgearbeitet hat, wie u. a. sein Sendschreiben an SICKLER in Band 2 beweist. Von Band 5 der "Curiositäten», in dem Sicklers Abhandlung über Puteoli erschienen ist, läßt sich sogar nachweisen, daß ihn GoETHE gekannt hat; er hat ihn nämlich vom 19. bis 27. April 1819 aus der Weimarer Bibliothek entlehnt gehabt ${ }^{10}$. Auch da Hoff gerade an jener Stelle seines Werkes, an welche GoETHE anknüpfte, sich auf SICKLER bezieht, darf angenommen werden, daß GOETHE dessen Abhandlung gelesen hat, und sei es auch erst auf Grund von Hoffs Zitat gewesen.

Selbst wenn GOETHE von SiCKLER als Kunstarchäologen nicht viel gehalten zu haben scheint, hätte er als Naturforscher nicht über dessen Hinweis auf die weitere Verbreitung der Muschellöcher hinweggehen dürfen. Er heftete in diesem Falle den Blick aber nur auf eine einzige Erscheinung und unterließ die Nachprüfung, ob es ähnliche auch anderwärts gibt und ob auch dort die gleiche Erklärung anwendbar ist. Dazu kommt, daß er sich, allzu souverän, auf Streit und Widerstreit nicht einlassen mochte, und daß er, der überall die Harmonie suchte, den Gedanken an Landhebungen als gewaltsam und unharmonisch ablehnte.

Beim Serapäum sah er infolge der Schuttmassen und des Bächleins die Möglichkeit der Entstehung eines Teiches gegeben, und in diesen versetzte er die Bohrmuscheln. Negativ führte er für seine Auffassung an, daß es keine literarische Nachricht über eine ehemalige Meereshebung gebe. Er bezeichnet seine Erklärung als lokal, womit er wohl sagen will, daß sie nicht auf einem durch Beobachtungen anderwärts entstandenen und auf Puteoli übertragenen Schlusse beruhe, und er nennt sie als «mit dem geringsten Aufwande die Sache zur Klarheit» bringend. Seine Erklärung ist gewißlich lokal, und sie beansprucht tatsächlich den geringsten Aufwand, weil sie nur ein einziges Naturereignis voraussetzt, die Verschüttung des Tempels und darauffolgende Teichbildung, während wir nach der Verschüttung noch zwei andere gewaltsame Vorgänge annehmen, die Senkung der Küste und deren spätere Hebung. Aber Goethe mußte doch den anderen Aufwand hinnehmen, daß er den Bohrmuscheln Lebensmöglichkeit in Süßwasser zuschrieb, was gegen jede Erfahrung ist.

${ }^{10}$ E. v. Keudell: Goethe als Benützer der Weimarer Bibliothek (Weimar 1931), S. 197, Nr. 1232. 
Wie früher erwähnt, muß GoEthe das Vorkommen von Bohrmuscheln anderwärts als nur in Pozzuoli ebenfalls gekannt haben, wenn auch vielleicht nur auf literarischem Wege. Dennoch hat er sich auf seine «lokale Erklärung» versteift.' Dieser Eigensinn, der als unwissenschaftlich bezeichnet werden muß, weil er GOETHE davon abgehalten hat, alle in Betracht kommenden Erscheinungen unvoreingenommen heranzuziehen, erklärt sich aus seinem Abscheu vor der Annahme gewaltsamer Naturvorgänge. Den in dieser Beziehung aufschlußreichen, oben Seite 208 wiedergegebenen Äußerungen GOETHEs sei noch angefügt, daß er in seinen "Annalen» 1820 anläßlich der Erwähnung seines Studiums eines Granitgebirges auf der Reise über Wunsiedel nach Alexandersbad seinen «Abscheu vor gewaltsamen Erklärungen ... mit reichlichen Erdbeben, Vulkanen, Wasserfluten und anderen titanischen Ereignissen» ausgesprochen hat.

Was in Goethes Augen gewaltsame und desperate Erklärungen waren, wie die Hebung und das Sinken ganzer Landmassen, mit der die moderne Geologie noch stärker rechnet als die zu GoETHEs Zeit, erscheint weniger gewaltsam, wenn man bedenkt, daß die feste Erdrinde, auf deren Oberfläche sich die betreffenden Vorgänge vollziehen, nur eine dünne Schale auf der Erdkugel ist, etwa $100 \mathrm{~km}$ dick gegenüber den $6378 \mathrm{~km}$ des Äquatordurchmessers der Erde. Wie sehr selbst die Hochgebirge in der Erdmasse verschwinden, wird klar, wenn man weiß, daß die über $18 \mathrm{~km}$ betragenden Höhenunterschiede der Erdoberfläche - größte absolute Höhe im Himalaja über $8800 \mathrm{~m}$, größte gelotete Ozeantiefe über $9700 \mathrm{~m}$ - auf einem Globus von $1 \mathrm{~m}$ Durchmesser nur ungefähr $3 \mathrm{~mm}$ ausmachen.

GoEthes Aufsatz ist also für seine eigenwillige und irrige Auffassung gewisser geologischer Vorgänge bezeichnend, aber auch für seine überaus stark naturkundliche Einstellung. Schon 1787 zogen ihn die Bohrmuschellöcher an, und auch 1823 stellte er all die bau- und kunstgeschichtlichen sowie die religionsgeschichtlichen Fragen, die sich an das Serapäum knüpfen lassen, durchaus in den Hintergrund zugunsten des naturwissenschaftlichen Problems.

Sowohl Goethe als auch Sickler haben sich Gedanken über den Zeitpunkt gemacht, an dem die Bohrmuscheln ihre Tätigkeit in den Säulen des Serapäums ausgeübt haben. SiCKLER waren durch Angaben anderer Altertumsforscher und durch eigene Beobachtungenantike Gräber in Kampanien bekannt, die in einer Schicht aus Sand und zerriebenem Bimsstein staken, über sich zunächst eine Humusschicht, dann eine Sand- und Bimssteinschicht, zuoberst schließlich wieder Humus. Da die beiden oberen Schichten über Gräbern mit "campanischen» Vasen, wie SrCKLER die bemalten griechischen Tongefäße Unteritaliens nennt, oft ungestört angetroffen werden, müssen sie so hatte schon der von SiCKLER zitierte Abbate Mazzuola geschlossen - nach dem Herrichten der betreffenden Gräber entstanden sein, weil sie sonst verwühlt worden wären. Die von oben her als zweite Lage anzutreffende Schicht aus Sand, Kies und Bimssteinen sei nur in der Ebene vorhanden, nicht auch auf den Höhen, sie müsse daher eine Meeresablagerung sein und dieses müsse mithin nach dem Bau der Gräber die Ebene überflutet haben.

Hatte SICKLER auf diese Weise aus den Bodenschichten einen relativen terminus ante quem gewonnen, so ergab sich ihm ein weiterer aus Mazzuolas und seiner eigenen Beobachtung, daß griechische bemalte Keramik in Pompeji, Herculaneum und Stabiae nicht gefunden wurde und $\mathrm{da} \beta$ auch die Wandgemälde in Pompeji keine Vasen zeigten, «die eine nur entfernte Ähnlichkeit mit den echten kampanischen Vasen von weitem verraten».

SICKLER erörtert dann die von Bohrmuscheln erzeugten Löcher an Küstenfelsen und in antiken Baudenkmälern als weiteren Beweis für eine alte Meerestransgression, und er stellt die Frage nach deren Datierung. Auf Grund einer antiken Nachricht setzt er den Beginn der römischen Besiedlung der Gegend von Pästum ins Jahr 264 v. Chr. Da die römischen Baureste in dieser Gegend keine Spur von Bohrmuscheln aufweisen, 
ebenso nicht die «echtrömischen Monumente» bei Baiae, die "mit ihrem Grunde der Meeresoberfläche gleichstehen", "während die Felsen über dem Meere, Puzzuolis und Pästums dorische Säulen überall deutlich davon zeugen", schließt er, daß die große Uberflutung vor 264 stattgefunden haben müsse und nach dem 6 . Jahrh. v. Chr., weil in letzterem Unteritalien als besonders fruchtbar geschildert worden sei, mithin damals noch nicht von Meeressand bedeckt gewesen sein könne.

SickLers Erwägungen fallen deswegen auf, weil man sich zu seineı Zeit mit Datierungsfragen nur am Rande und gelegentlich abgegeben hat; man war sich weder dessen voll bewußt, daß für jede kunstgeschichtliche, also nicht nur ästhetische Betrachtung die Datierung eines Altertums unerläßlich ist, noch auch hatte man überhaupt Methoden, um Datierungsproblemen mit Aussicht auf Erfolg zu Leibe gehen zu können. SicKLER macht hier insofern eine rühmenswerte Ausnahme, als er durch das Kombinieren von Altertümern mit naturkundlichen Beobachtungen eine chronologische Basis errichten wollte.

Seine Schlüsse sind allerdings nicht durchwegs bündig. Die Tatsache nämlich, daß nicht alle antiken Baureste Löcher von Bohrmuscheln aufweisen, braucht an sich noch nichts mit dem Datum ihrer Überflutung zu tun haben, sondern kann auch darauf zurückzuführen sein, daß nicht alle Küstengewässer den Bohrmuscheln zugesagt haben müssen; diese können ein örtlich ganz verschiedenes Auftreten haben. Weiters scheinen die Bohrmuscheln nicht jedes Gestein anzugreifen. Die Pästum-Tempel, von denen die Basilica und der Ceres-Tempel aus dem 6. Jahrh. v. Chr. stammen, der PoseidonTempel aus dem 5. Jahrh. v. Chr., bestehen aus Tuff. Seit der Zeit des Augustus haben die Baumeister Marmor als Werkstoff vorgezogen, und dieser ist meist härter als Tuff, was die Bohrmuscheln leicht abschrecken kann. Unter den verschiedenen Marmorsorten gibt es freilich verschiedene Härten; der Marmor der Säulen des Serapäums scheint ein weicherer zu sein.

Die Frage, wann das Serapäum unter Wasser geraten ist, hat auch den englischen Geologen C. LyeLL in seinem Buche "Principles of Geology» beschäftigt ${ }^{11}$. Eingehendes Studium der Küstengegend, der archäologischen Überreste und alter Nachrichten ließen ihn zu folgendem Ergebnis kommen:

«Ungefähr $80 \mathrm{Jahre}$ vor der christlichen Zeitrechnung, als der alte Mosaikfußboden» (auf den man durch eine Ausgrabung im Jahre 1828 gestoßen war) «angefertigt wurde, lag derselbe ungefähr $12 \mathrm{Fuß}$ über dem jetzigen Meeresspiegel oder über seinem Stand von 1838. Gegen den Schluß des 1. Jahrh. n. Chr. lag er ungefähr $6 \mathrm{Fuß}$ über seinem jetzigen Niveau. Am Ende des 4. Jahrh. war er fast bis.zu seinem jetzigen Niveau herabgesunken. Im Mittelalter und vor dem Ausbruche des Monte Nuovo" (im Jahre 1538) «lag er ungefähr $19 \mathrm{Fuß}$ unter seinem jetzigen Niveau. Endlich zu Anfang des jetzigen Jahrhunderts» (also des 19.) «lag er etwa 26 Zoll über seinem Stande im Jahre 1838." LyelL fügt hinzu, BABBAGE sei auf anderem Wege zur Úberzeugung gelangt, da $ß$ das Niedersinken des Tempels stufenweise erfolgte und so lange andauerte, bis der Boden zuletzt $15 \mathrm{Fuß}$ unter dem Meeresspiegel lag. Als Ursache für das Niedersinken des Gebäudes und seine Hebung erkannte LyELL Landbe wegungen, nicht Steigen und Fallen des Meeres.

Eine Erklärung des Phänomens von Pozzuoli, die von der Lyelzs und Goethes gänzlich abweicht, hat der Professor für technische Geologie und Bodenkunde an der Universität Halle, D. Brauns, geboten ${ }^{12}$. Er faßte das Serapäum als Bassin für vorrätig gehaltene Seetiere auf. In dem Bassin sei Seewasser bis zu einer gewissen Höhe gestanden, und da die Bohrmuscheln, die entweder zufällig in das Bassin geraten seien oder die man dort gezogen habe, sich nahe der Wasseroberfläche aufhalten, sei die Anbohrung der Säulen durch sie in einer bestimmten Höhe erklärlich. BRAUNS sagt von seiner

11 Von diesem Werk liegt mir die nach der 6. Originalauflage besorgte deutsche Übersetzung von C. Hartmann vor, wo sich die Ausführungen über das Serapäum in Band 2 (Weimar 1841), S. 530-547, finden.

12 D. Brauns: Das Problem des Serapäums von Pozzuoli (Leopoldina 24, Halle 1888, S. 132, 150, $161,189,209)$. 
Auffassung, sie befreie uns "von der durchaus unhaltbaren Annahme einer Landsenkung und Wiederhebung".

Auch Brauns schien also, so wie Goethe, der Gedanke an Hebung und Senkung des Landes unannehmbar. Heute rechnet mit ihm die Erdkunde ohne Widerstreben, selbst dann, wenn durch die Landbewegung auch Bauwerke betroffen worden sind; LYELL bringt aus verschiedenen Teilen der Welt Beispiele dafür, daß Gebäude unter Wasser gesetzt und später wieder gehoben wurden, ohne in einen Trümmerhaufen verwandelt worden zu sein. Unter den Belegen für Senkung und Hebung von Landstrecken führt F. X. SCHAFFRR: Grundzüge der allgemeinen Geologie I (Leipzig 1916), S. 105 ff., als den schönsten gerade das Serapäum von Pozzuoli an ${ }^{13}$.

GOETHE hat von Art und Ausdehnung der geologischen Vorgänge an den Mittelmeerküsten unzutreffende Auffassungen gehabt, und auch SICKLER war nicht auf dem richtigen Wege, als er nur mit Steigen des Meeresspiegels rechnete.

GoETHE hat die Annahme von Landhebungen abgelehnt, wobei er sich, wie wir gesehen haben, geradezu erbittert ausgedrückt hat. In seiner Auffassung macht sich schließlich wiederum der ihn stets und überall beherrschende Entwicklungsgedanke geltend. Entwicklung bedeutete für GOETHE nicht sprunghafte, vor allem nicht gewaltsame Änderung von Bestehendem, sondern organisches, langsam-stetiges Übergehen des einen Zustandes in den anderen. Landhebungen konnten ihm daher als etwas Gewaltsames ein abstoßender Gedanke sein; er projizierte also seinen eigenen geistigen Werdegang in die Natur; denn auch dieser zeichnet sich durch das Fehlen von Gewaltsamem, Abruptem aus. Freilich hat GOETHE bei seiner Stellungnahme gegenüber den erwähnten geologischen Vorgängen übersehen, daß Landhebungen zwar dem Menschen im Mikrokosmos als etwas Gewaltsames erscheinen können, daß sie aber im riesenhaften Haushalt der Natur Geschehnisse sind, die immer noch in den Bereich der organischen Entwicklung gehören, weil sie, im großen gesehen und ohne Rücksicht auf ihre Auswirkung für das Menschenleben, sinnvoller Ausfluß vorhergehender Zustände sind. Seine Ansicht über das Serapäum ist daher als Zeugnis für sein harmonisches Denken ehrwürdig, wenngleich sie als überholt zu gelten hat.

\section{GETHE ET LES CHANGEMENTS COTIERS PRES DE NAPLES}

Selon Gœthe, les trous percés par un genre de moules dans les colonnes du Sérapéum antique à Pozzuoli résultent du fait, que de telles moules vivaient dans un étang qui s'était formé à l'intérieur de cet immeuble. D'après d'autres opinions, les moules s'attachaient aux colonnes lors du rehaussement du niveau de la mer. Gœthe fut un antagoniste aussi bien de la théorie du rehaussement maritime que de celle de mouvements terrestres. Son opinion s'explique par son image du monde harmonique, défavorable à toute évolution violente. Sa conception relative au Sérapéum, en tant que témoignage, est encore aujourd'hui vénérable, bien qu'elle doit être considérée comme dépassée.

\section{GOETHE E LE VARIAZIONI DELLA COSTA PRESSO NAPOLI}

Goethe pensò che $\mathrm{i}$ fori osservati nelle colonne dell'antico tempio Serapeo di Pozzuoli fossero stati originati da lamellibranchi, i quali vissero in uno stagno formatosi fra le rovine del tempio stesso e non a lamellibranchi marini arrivati fino alle colonne in conseguenza a un inalzamento del livello del mare oppure a un abbassamento della crosta terrestre come veniva ritenuto da altri. Il poeta prese posizione contro queste interpretazioni perchè non poteva ammettere che nell'armonicità dell'evoluzione del mondo potessero intervenire fenomeni cosi violenti. L'interpretazione di Goethe è ora superata ma resta a testimoniare lodevolmente il suo pensiero.

13 Abb. 121 bei Schaffer gibt die Säulen nach LyelL; man sieht den Boden des Gebäudes von Wasser bedeckt; Abb. 122 ist eine Photographie aus dem Jahre 1896, der Tempelboden ist trocken. Für die neuern Untersuchungen vgl. E. Majo: Il bradisisma flegreo. Ann. Osservatorio Vesuviano, Reihe 4, Bd. II, 1929/30 (1934), S. 261-273. 